\title{
Neonatal exposure to sevoflurane causes apoptosis and reduces nNOS protein expression in rat hippocampus
}

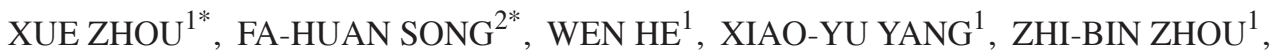 \\ $\mathrm{XIA} \mathrm{FENG}^{1}$ and LI-HUA $\mathrm{ZHOU}^{2}$ \\ ${ }^{1}$ Department of Anesthesiology, First Affiliated Hospital of Sun Yat-sen University; \\ ${ }^{2}$ Department of Anatomy, Zhong Shan School of Medicine, Sun Yat-sen University, \\ Guangzhou, Guangdong 510080, P.R. China
}

Received March 31, 2012; Accepted June 15, 2012

DOI: $10.3892 / \mathrm{mmr} .2012 .976$

\begin{abstract}
A growing number of studies have shown that commonly used anesthetic agents may cause neurohistopathological changes and persistent behavioral impairments in the developing brain. The effects of sevoflurane, a widely used substance in pediatric anesthesia, on the developing brain have not been thoroughly analyzed thus far. In this study, Sprague-Dawley rats at postnatal day $(\mathrm{P}) 7$ were exposed to $2.3 \%$ sevoflurane for $6 \mathrm{~h}$ continuously. Six hours after exposure, hematoxylin and eosin (H\&E) staining was used to determine the morphological changes in the hippocampus. The expression levels of neuronal nitric oxide synthase (nNOS) and caspase-3 in the hippocampus of the pups were determined by immunofluorescence. A single 6-h sevoflurane exposure at P7 caused morphological changes in the hippocampus. Sevoflurane also significantly increased the expression of cleaved caspase-3 and reduced the expression of the nNOS protein. These results indicate that neonatal exposure to sevoflurane causes neurohistopathological changes, apoptosis and decreases nNOS protein levels in the rat hippocampus.
\end{abstract}

\section{Introduction}

Due to the advancements in pediatric surgery, the chance for neonatal exposure to anesthetics has increased. Previous experimental studies have suggested that early exposure to anesthetic

Correspondence to: Dr Xia Feng, Department of Anesthesiology, First Affiliated Hospital of Sun Yat-sen University, 58 Zhongshan 2nd Road, Guangzhou, Guangdong 510080, P.R. China

E-mail: fengxiar@yahoo.cn

Professor Li-Hua Zhou, Department of Anatomy, Zhong Shan School of Medicine, Sun Yat-sen University, 74 Zhongshan 2nd Road, Guangzhou, Guangdong 510080, P.R. China

E-mail: zhoulih@mail.sysu.edu.cn

*Contributed equally

Key words: sevoflurane, neuronal nitric oxide synthase, apoptosis, hematoxylin and eosin staining agents, prior to the completion of synaptogenesis, may result in widespread apoptotic neuronal degeneration and late learning disability (1-3). Therefore, it is necessary to examine the effects of anesthetics on neuronal apoptosis associated with neurohistopathologic changes. All clinically used general anesthetics enhance GABAA receptors, block N-methyl-d-aspartate (NMDA) receptors, or both. In contrast to the mature brain, however, it has recently been discovered that the transient pharmacological blockade of NMDA receptors in the developing rodent brain causes excessive neuronal apoptosis (4).

Sevoflurane, one of the most frequently used volatile anesthetics, is particularly useful for infants and children, as it allows for rapid induction and recovery, and is less irritative to the airway (5). Sevoflurane has been shown to enhance GABAA receptors (6) and block NMDA receptors (7). Although there are certain studies that have demonstrated through in vivo and in vitro experiments that sevoflurane may effect cell survival and potentiate neuronal apoptosis $(8,9)$, studies on the effect of sevoflurane on the hippocampus of the developing brain are yet to be conducted.

In the brain, nitric oxide (NO), produced mainly by neuronal nitric oxide synthase (nNOS), behaves as an intercellular and intracellular diffusible messenger involved in multiple functions from developmental neural plasticity to the control of neurotransmitter release and memory consolidation $(10,11)$. Studies have demonstrated that the sustained inhibition of NO production triggers apoptosis in differentiated cerebellar granule neuron cultures (12). NO seems to have distinct functions during the different stages of ontogenesis of the forebrain, midbrain and cerebellum in rats (13), which is also reflected in the nNOS expression.

Caspases are crucial mediators of programmed cell death (apoptosis). Among them, caspase-3 is a frequently activated death protease, catalyzing the specific cleavage of many key cellular proteins (14). In multiple cell types, caspase-3 is required for certain typical nuclear and other morphological changes associated with the completion of apoptosis and the formation of apoptotic bodies (15-17).

In this study, to investigate the possible neurotoxicity induced by sevoflurane, we exposed neonatal rats to sevoflurane and assessed the morphological changes, as well as the expression of nNOS and cleaved caspase-3 in the hippocampus. 


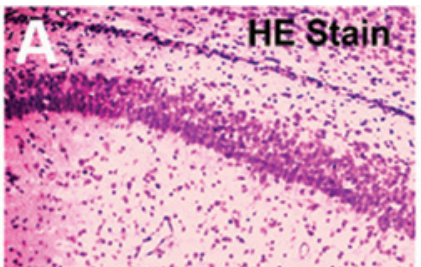

Air dontroi:
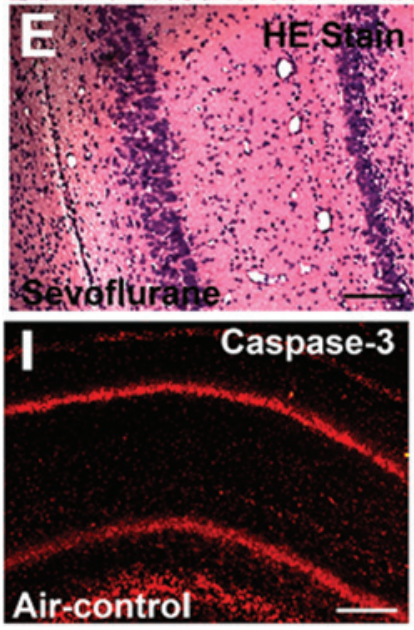

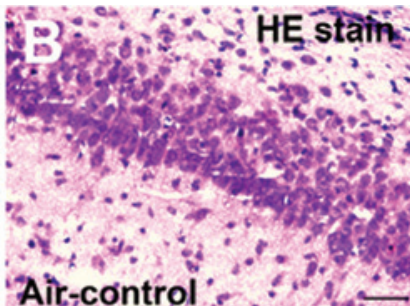

Ailt-control
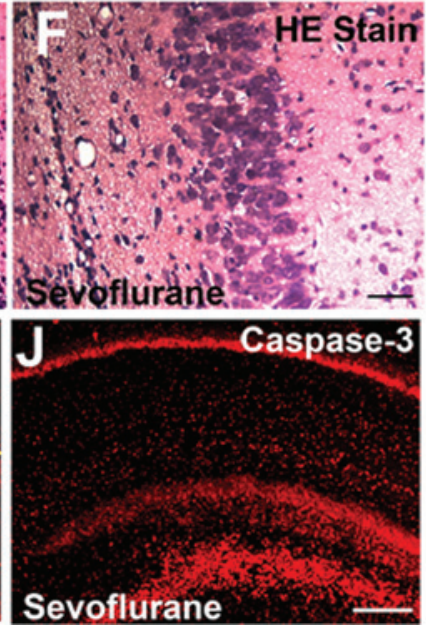
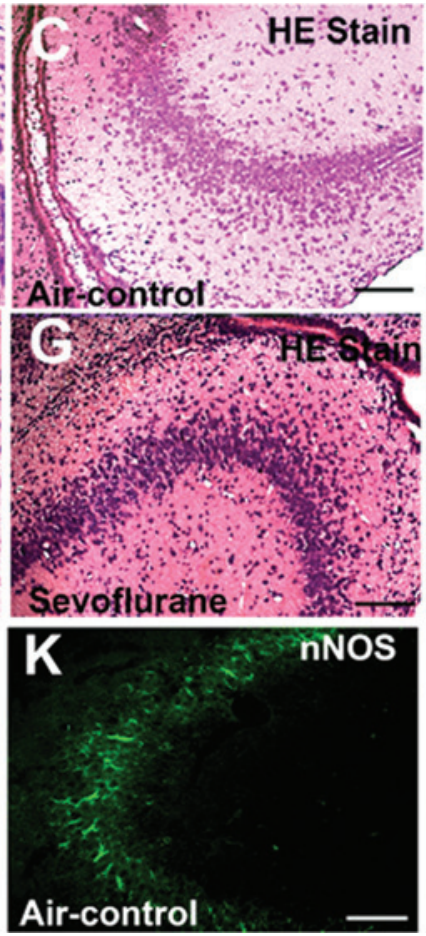
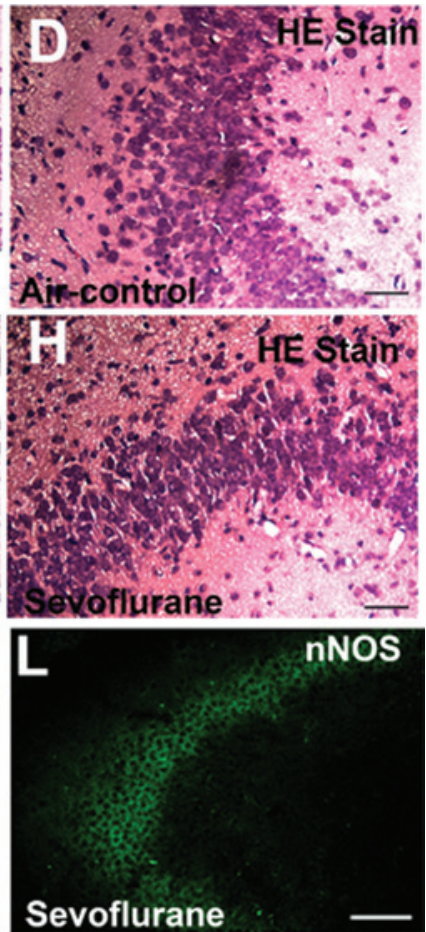

Figure 1. Sevoflurane inhalation caused morphological changes in parts of the CA1 and CA3 regions, and it also significantly activated caspase-3 and decreased neuronal nitric oxide synthase (nNOS) levels in both CA1 and CA3 regions $6 \mathrm{~h}$ after exposure. (A-D) Control group; (E-H) sevoflurane group. (A, B, E and F) Low-magnification images of the $\mathrm{CA} 1$ region (A and $\mathrm{E}$ bar, $100 \mu \mathrm{m}$ ) and high-magnification images of the $\mathrm{CA} 1 \mathrm{region}(\mathrm{B}$ and $\mathrm{F}$ bar, $20 \mu \mathrm{m})$. (C, $\mathrm{D}, \mathrm{G}$ and $\mathrm{H})$ Low-Magnification images of the CA3 region (C and $\mathrm{G}$ bar,100 $\mu \mathrm{m}$ ) and high-magnification images of the CA3 region (D and $\mathrm{H}$ bar, $20 \mu \mathrm{m})$. Morphological changes in the sevoflurane group can be observed. In the sevoflurane group (E-H), the cells were disorganized and not that closely ranked, and some nuclei were not in the center of the eosinophilic cells. (I-L) nNOS (green) and caspase-3 (red) immunofluorescence staining of the hippocampus at the 6-h point after treatment. (I) CA1 field of the control group; (J) CA1 field of the sevoflurane group; (K) CA3 field of the control group; (L) CA3 field of the sevoflurane group. The images show that the nNOS-positive cells in the sevoflurane group were less than the nNOS-positive cells in the control group, in both the CA1 and CA3 fields. The images also show that caspase-3 levels in the sevoflurane group were more increased compared to the control group in the CA1 field. (I-L bar, $100 \mu \mathrm{m}$ ).

\section{Materials and methods}

Animals. Male Sprague-Dawley (SD) rats obtained from the Experimental Animal Center of the Sun Yat-sen University Guangzhou, China, were used in this study. The use of aimals in this study was approved by the Institutional Animal Care and Use Committee of Sun Yat-sen University. All efforts were made to minimize the number of animals used as well as their suffering. The room was illuminated with a 12-h lightdark cycle (light from 07:00-19:00), and the room temperature was maintained at $21 \pm 1^{\circ} \mathrm{C}$.

Sevoflurane exposure. The SD rats at postnatal day (P)7 (weight, 16-17 g) were randomly divided into an air-treated control group and a sevoflurane-treated group. Rats in the sevoflurane group were placed in a plastic container and exposed to $2.3 \%$ sevoflurane for $6 \mathrm{~h}$ continuously, using air as a carrier with a total gas flow of $2 \mathrm{l} / \mathrm{min}$. During the sevoflurane exposure, the container was heated to $38^{\circ} \mathrm{C}$ using a heating device (NPS-A3 heated device; Midea Co., Guangdong, China). The levels of sevoflurane, oxygen and carbon dioxide were monitored in the chamber, using a gas monitor (Detex-Ohmeda, Louisville, CO, USA). Sevoflurane administration was terminated $6 \mathrm{~h}$ later and the rats were exposed to air solely. When the rats were once again moving freely, they were placed back into the maternal cage. During exposure to sevoflurane, the respiratory frequency and skin color of the rats were monitored. In case of apnea or hypoxia, the rat was immediately exposed to air and excluded from the experiment. Rats (at P7) in the control group were placed into the same container as the rats in the sevoflurane group, but were exposed to air alone for $6 \mathrm{~h}$.

Histopathological examination. Sevoflurane-exposed rats as well as rats from the control group (16-17 g) were sacrificed at $6 \mathrm{~h}(\mathrm{n}=3)$, after a 6 -h exposure. Tissue blocks $(0.5 \mathrm{~cm}$ thick) from the hippocampus were embedded in paraffin, sliced in 5-mm-thick sections and stained with hematoxylin and eosin (H\&E). The results were examined in detail under a light microscope so as to determine morphological changes in parts of the $\mathrm{CA} 1$ and $\mathrm{CA} 3$ regions.

Immunofluorescence. Sevoflurane-exposed rats as well as rats (16-17 g) from the control group ( $n=3 /$ group) were deeply anesthetized with chloral hydrate at $6 \mathrm{~h}$ after a 6 -h exposure, and then perfused with $4 \%$ paraformaldehyde (Sigma-Aldrich, St. Louis, MO, USA) in PBS (pH 7.4) through the left cardiac ventricle, in order to assess the sevoflurane exposure-induced changes on nNOS and caspase-3 levels in the hippocampus. Brains were dissected out and placed in the same fixative solution overnight. After postfixation, the brains were soaked in $30 \%$ sucrose for an additional $24 \mathrm{~h}$.

Coronal sections $(20 \mu \mathrm{m})$ were cut using a sliding microtome (Scientific Instruments, Palm Beach, FL, USA) and subsequently processed for immunofluorescence analysis. Briefly, 
the floating sections were blocked with a solution containing $1 \%$ BSA and $0.4 \%$ Triton X-100 for $2 \mathrm{~h}$ at room temperature and incubated with mouse anti-nNOS (diluted 1:3000; Santa Cruz Biotechnology, Inc., Santa Cruz, CA, USA) and rabbit cleaved caspase-3 (diluted 1:15000; Cell Signaling Technology, Inc., Danvers, MA, USA) at $4^{\circ} \mathrm{C}$ overnight. After washing with PBS, the sections were incubated with anti-mouse IgG tetrarhodamine isothiocyanate (TRITC) conjugate (diluted 1:800; Sigma-Aldrich) and anti-rabbit IgG FITC conjugate (diluted 1:400; Sigma-Aldrich) in the dark. Finally, the sections were rinsed with PBS, mounted on gel-coated slides and observed under a microscope (Axio Imager Z1). Each experiment was repeated independently at least 3 times.

\section{Results}

Inhalation of sevoflurane for $6 \mathrm{~h}$ decreases the number of eosinophilic cells and causes morphological changes in parts of $C A 1$ and $C A 3$ regions. In the control group, eosinophilic cells in the CA1 region were arranged in neat order and observed under a microscope (Fig. 1A). At high magnification, at least 5 layers of cells with cell size uniformity were detected (Fig. 1B). In the sevoflurane group, the cells in the CA1 region were arranged in a disorganized manner and were not so closely attached (Fig. 1E). At high magnification, several dark neurons with destroyed nuclei were observed. Moreover, the number of the cell layers decreased (Fig. 1F). In the control group, a dense radiating cluster of eosinophilic cells in the CA3 region were arranged in neat order (Fig. $1 C$ and D). In the sevoflurane group, however, morphological changes could be observed, while in the CA3 region various dark neurons were detected. Some nuclei were not in the center of the eosinophilic cells, indicating that apoptosis may occur due to sevoflurane inhalation (Fig. $1 \mathrm{G}$ and $\mathrm{H}$ ).

Exposure to sevoflurane-induces expression of cleaved caspase-3 and reduces expression of nNOS in neonatal rat hippocampus. The effects of sevoflurane on cleaved caspase-3 adn nNOS expression are presented in Fig. 1I-L. Both cleaved caspase-3 (Fig. 1I) and nNOS (Fig. 1K) were expressed in the hippocampus of the control rats. In the CA1 region of the hippocampus, cleaved-caspase immunostaining greatly increased at the 6-h point, after sevoflurane inhalation. nNOSpositive cells were found in the CA3 region of rats in the control group (Fig. 1K). However, after the 6-h exposure to sevoflurane, nNOS immunostaining decreased (Fig. 1L). The images show that nNOS-positive cells in the sevoflurane group were less than the nNOS-positive cells in the control group in the CA3 field.

\section{Discussion}

Due to the advances in fetal surgery, there is an increase in the duration and complexity of anesthesia. Sevoflurane is particularly useful for infants and children, as it allows for rapid induction and recovery, and is less irritative to the airway (5). There is evidence indicating that exposure to sevoflurane results in apoptosis in the developing brain (18). The hippocampus is part of the limbic system of the brain, highly associated with neuronal synaptic plasticity, learning and memory functions, and it is easily damaged due to its structure (19). In the present study, we selected $2.3 \%$ sevoflurane, as this was the highest concentration not inhibiting respiration and circulation in rat pups under our experimental conditions and was comparable to the concentration used in clinical settings.

In our study, H\&E staining results demonstrated that a single 6-h sevoflurane exposure at P7 caused morphological changes in the hippocampus. Compared with the air-control groups both in the CA1 and CA3 fields, the cells were disorganized and not that closely attached. Under high magnification it was observed that in the sevoflurane group, the nuclei in certain cells were not in the center of the cytoplasm.

The immunofluorescence results showed that exposure to sevoflurane induced the expression of cleaved caspase- 3 and reduced the expression of nNOS in the neonatal rat hippocampus. Caspase-3, when activated by proteolytic cleavage, is one of the apoptotic effectors responsible for the breakdown of cellular components. Activated caspase-3 is widely used as a marker for apoptotic cells (20). In our study, the results indicated that a 6 -h exposure to $2.3 \%$ sevoflurane induced apoptosis in the hippocampus. The nNOS is the predominant NOS isoform in the nervous system and can be transcriptionally induced under certain circumstances, such as neuronal development (21). In the brain, NO produced mainly by nNOS, plays an important role in central nervous system (CNS) functions, including apoptosis, neurogenesis, neuronal differentiation and development (22). We show that a 6-h exposure to $2.3 \%$ sevoflurane induces a decrease in nNOS levels, which may participate in neuronal apoptosis. However, additional investigations are required to determine whether this decrease will continue through to adulthood.

In conclusion, neonatal exposure to $2.3 \%$ sevoflurane for $6 \mathrm{~h}$ causes neurohistopathological changes, apoptosis and decreases nNOS protein levels in the rat hippocampus.

\section{Acknowledgements}

The authors gratefully acknowledge the financial support of the National Science Foundation Council of China (31140050), Guangdong Science Foundations (2010B031600037) and Guangdong Science and Technology Planning Project (2010B031600207; 2011B050400024).

\section{References}

1. Anand KJ: Anesthetic neurotoxicity in newborns: should we change clinical practice? Anesthesiology 107: 2-4, 2007.

2. Wiklund A, Granon S, Faure P, Sundman E, Changeux JP and Eriksson LI: Object memory in young and aged mice after sevoflurane anaesthesia. Neuroreport 20: 1419-1423, 2009.

3. Jevtovic-Todorovic V, Hartman RE, Izumi Y, et al: Early exposure to common anesthetic agents causes widespread neurodegeneration in the developing rat brain and persistent learning deficits. J Neurosci 23: 876-882, 2003.

4. Perouansky M: General anesthetics and long-term neurotoxicity. Handb Exp Pharmacol 182: 143-157, 2008.

5. Lerman J, Sikich N, Kleinman S and Yentis S: The pharmacology of sevoflurane in infants and children. Anesthesiology 80: 814-824, 1994.

6. Shelton KL: Discriminative stimulus effects of inhaled 1,1,1-trichloroethane in mice: comparison to other hydrocarbon vapors and volatile anesthetics. Psychopharmacology (Berl) 203: 431-440, 2009. 
7. Nishikawa $\mathrm{K}$ and Harrison NL: The actions of sevoflurane and desflurane on the gamma-aminobutyric acid receptor type A: effects of TM2 mutations in the alpha and beta subunits. Anesthesiology 99: 678-684, 2003.

8. Kvolik S, Dobrosevic B, Marczi S, Prlic L and Glavas-Obrovac L: Different apoptosis ratios and gene expressions in two human cell lines after sevoflurane anaesthesia. Acta Anaesthesiol Scand 53: 1192-1199, 2009.

9. Dong Y, Zhang G, Zhang B, Moir RD, Xia W, Marcantonio ER, Culley DJ, Crosby G, Tanzi RE and Xie Z: The common inhalational anesthetic sevoflurane induces apoptosis and increases beta-amyloid protein levels. Arch Neurol 66: 620-631, 2009.

10. Porro A, Chrochemore C, Cambuli F, Iraci N, Contestabile A and Perini G: Nitric oxide control of MYCN expression and mult drug resistance genes in tumours of neural origin. Curr Pharm Des 16: 431-439, 2010

11. Hsu YY, Liu CM, Tsai HH, Jong YJ, Chen IJ and Lo YC: KMUP-1 attenuates serum deprivation-induced neurotoxicity in SH-SY5Y cells: roles of PKG, PI3K/Akt and Bcl-2/Bax pathways. Toxicology 268: 46-54, 2010.

12. Ciani E, Guidi S, Della Valle G, Perini G, Bartesaghi R and Contestabile A: Nitric oxide protects neuroblastoma cells from apoptosis induced by serum deprivation through cAMP-response element-binding protein (CREB) activation. J Biol Chem 277: 49896-49902, 2002

13. Iwase K, Takemura M, Shimada T, Wakisaka S, Nokubi T and Shigenaga Y: Ontogeny of NADPH-diaphorase in rat forebrain and midbrain. Anat Embryol (Berl) 197: 229-247, 1998.

14. Porter AG and Janicke RU: Emerging roles of caspase-3 in apoptosis. Cell Death Differ 6: 99-104, 1999.
15. Woo M, Hakem R, Soengas MS, Duncan GS, Shahinian A, Kägi D, Hakem A, McCurrach M, Khoo W, Kaufman SA, et al: Essential contribution of caspase 3/CPP32 to apoptosis and its associated nuclear changes. Genes Dev 12: 806-819, 1998.

16. Hirata H, Takahashi A, Kobayashi S, Yonehara S, Sawai H, Okazaki T, Yamamoto K and Sasada M: Caspases are activated in a branched protease cascade and control distinct downstream processes in Fas-induced apoptosis. J Exp Med 187: 587-600, 1998.

17. Janicke RU, Sprengart ML, Wati MR and Porter AG: Caspase-3 is required for DNA fragmentation and morphological changes associated with apoptosis. J Biol Chem 273: 9357-9360, 1998.

18. Satomoto M, Satoh Y, Terui K, Miyao H, Takishima K, Ito M and Imaki J: Neonatal exposure to sevoflurane induces abnormal social behaviors and deficits in fear conditioning in mice. Anesthesiology 110: 628-637, 2009.

19. Win-Shwe TT, Yoshida Y, Kunugita N, Tsukahara S and Fujimaki H: Does early life toluene exposure alter the expression of NMDA receptor subunits and signal transduction pathway in infant mouse hippocampus? Neurotoxicology 31: 647-653, 2010.

20. Young C, Roth KA, Klocke BJ, West T, Holtzman DM, Labruyere J, Qin YQ, Dikranian K and Olney JW: Role of caspase-3 in ethanol-induced developmental neurodegeneration. Neurobiol Dis 20: 608-614, 2005

21. Knott AB and Bossy-Wetzel E: Nitric oxide in health and disease of the nervous system. Antioxid Redox Signal 11: 541-554, 2009.

22. Garthwaite J: Concepts of neural nitric oxide-mediated transmission. Eur J Neurosci 27: 2783-2802, 2008. 